
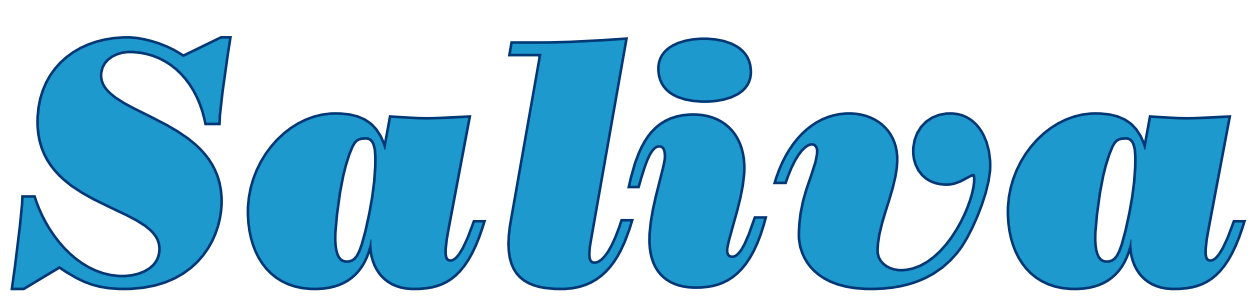

\title{
A review of its role in maintaining oral health and preventing dental disease
}

\section{by Michael Dodds, Senior Principal Technology Scientist at the Wrigley Company, with support from Simon Roland, Michael Edgar and Martin Thornhill.}

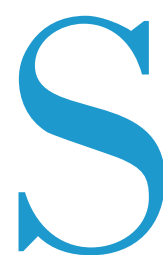

aliva plays a significant role in maintaining oral health, helping to build and maintain the health of soft and hard tissues. When saliva flow is reduced, oral health problems such as dental caries and oral infections can develop.

\section{Composition and production}

Saliva is an exocrine solution consisting of $99 \%$ water. The remaining $1 \%$ consists of a variety of electrolytes and proteins. ${ }^{1}$ These components combined are responsible for the various functions attributed to saliva. ${ }^{1}$

Saliva is formed primarily (approximately 90\%) from the secretions of the three paired major salivary glands, the submandibular (around 65\%), parotid (around 20\%) and sublingual (around 5-7\%). ${ }^{1}$ These glands are controlled by the autonomic nervous system, while minor glands (labial, lingual, buccal and palatine), distributed around the oral cavity, produce the remaining saliva $(<10 \%) .{ }^{1}$

At rest, without exogenous or pharmacological stimulation, there is a small, continuous salivary flow, an unstimulated secretion, present in the form of a film that covers, moisturises, and lubricates the oral tissues. ${ }^{1}$ This flow of saliva at rest is in the region of $0.4-0.5 \mathrm{~mL} /$ minute in healthy subjects. ${ }^{1}$

Stimulated saliva is produced in response to a mechanical, gustatory, olfactory, or pharmacological stimulus, contributing to around $40-50 \%$ of daily salivary production. ${ }^{2}$ The Salivary Flow (SF) index is a parameter allowing stimulated and unstimulated saliva flow to be classified as normal, low or very low (hyposalivation). In adults, normal total stimulated SF ranges $1-3 \mathrm{~mL} /$ minute, low ranges $0.7-1.0 \mathrm{~mL} /$ minute, while hyposalivation is characterised by a stimulated $\mathrm{SF}<0.7 \mathrm{~mL} /$ minute $^{1}$

\section{Key functions}

The two major functions of saliva are:

7. Protection of the oral and perioral tissues

- Lubrication

- Dilution of sugars after food and drink intake

- Antimicrobial and cleansing activity,

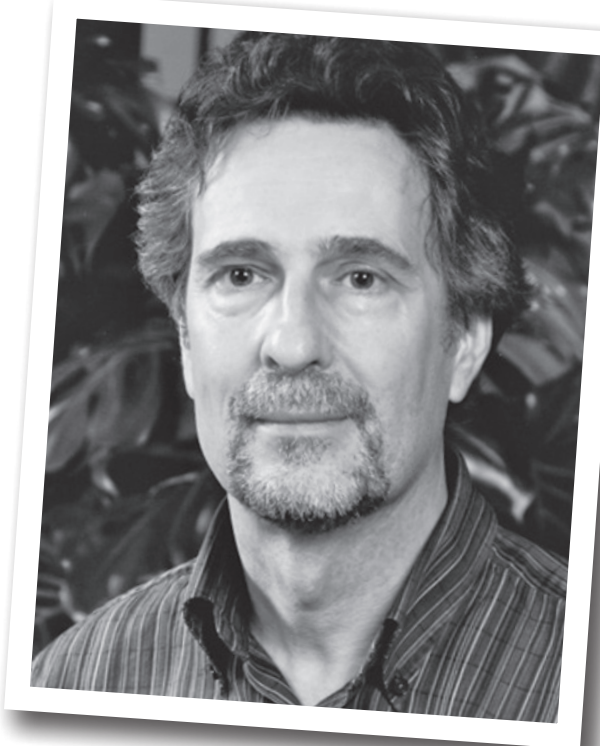

degrading some bacterial cell walls and inhibiting growth

- Buffering (neutralising) acid production and controlling plaque $\mathrm{pH}$ with bicarbonate

- Remineralisation of enamel with calcium and phosphates

- Tissue repair

\section{Figure 1: Factors affecting the development of dental caries}

Inside the mouth

- Bacterial composition of the biofilm

- Plaque pH

- Salivary flow rate (stimulated and unstimulated)

- Buffering effect of saliva

- Food retention

- Inorganic compounds $\left(\mathrm{Ca}^{2+}\right.$ and $\left.\mathrm{PO}_{4}{ }^{3-}\right)$

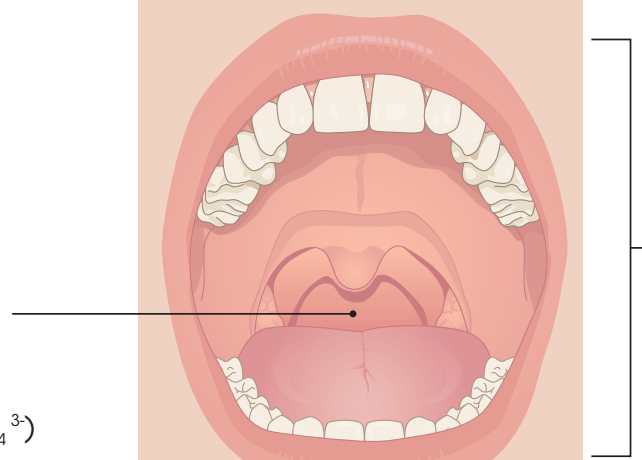

Outside the mouth

General health

- Medical history

- Hormones

- Age

- Genetic heritage

- Medical treatment
Environment

- Frequency of eating

- Diet

- Oral hygiene

- Fluoride 


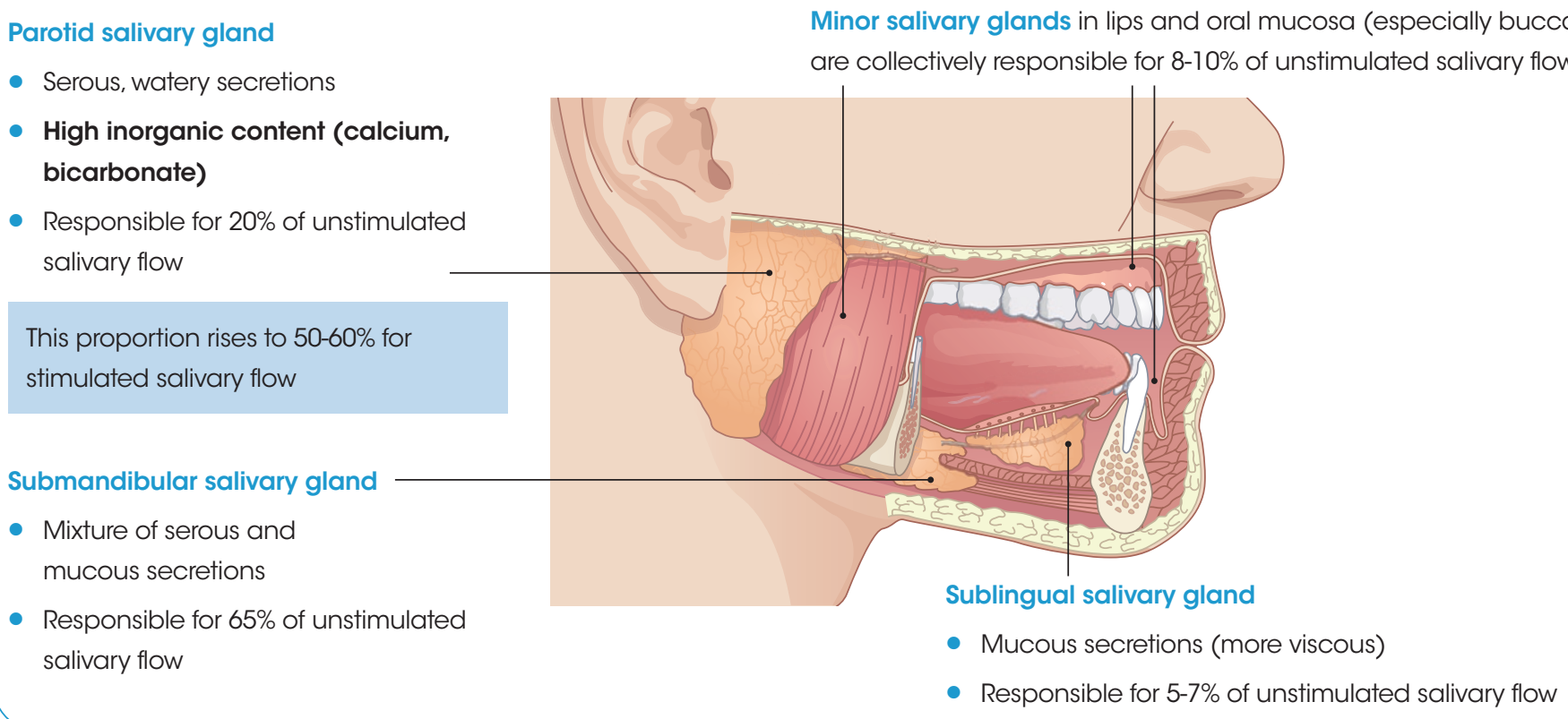

- Serous, watery secretions

High inorganic content (calcium, bicarbonate)

Responsible for $20 \%$ of unstimulated salivary flow

This proportion rises to $50-60 \%$ for stimulated salivary flow

Submandibular salivary gland

Mixture of serous and mucous secretion salivary flow

\section{Facilitating eating and speech}

- Food preparation, enhancing chewing, the clearing of food residues and swallowing

- Digestion, food breakdown with enzymes

- Enhancing taste

- Enabling speech by lubricating the moving oral tissues

\section{In addition, saliva is used in} diagnostic testing

- Bacterial, yeast, and viral counts indicating caries activity and altered immune responses, as well as many diagnostic tests for oral and systemic diseases

- Hormonal balance to identify steroids and sex hormones

\section{Saliva and dental caries}

In addition to moderating microbial factors and encouraging preventive dietary behaviours, a core goal in caries prevention is promoting the natural protective mechanisms of saliva. ${ }^{3}$

The $\mathrm{pH}$ of dental plaque is a key factor in the balance between acid demineralisation of the teeth and the remineralisation of the initial caries lesion. Plaque $\mathrm{pH}$ falls each time acid accumulates in the plaque due to bacterial acid production following the consumption of fermentable carbohydrates - mainly sugars - in foods and drinks. Conversely, plaque $\mathrm{pH}$ rises when the acids are washed away or neutralised by saliva, which contains the important buffer, bicarbonate.

In healthy teeth, the loss of minerals is balanced by the reparative mechanisms of

'STIMULATED SALIVA IS PRODUCED IN RESPONSE TO A MECHANICAL, GUSTATORY, OLFACTORY, OR PHARMACOLOGICAL STIMULUS, CONTRIBUTING TO

\section{AROUND 40-50\% OF DAILY SALIVARY PRODUCTION'}

saliva. This equilibrium can be depicted chemically by the equation overleaf - see Figure 3 opposite. $^{4}$

When the saliva $\mathrm{pH}$ or the plaque $\mathrm{pH}$ is below a 'critical value' of about 5.5 , the saliva or plaque becomes unsaturated with respect to tooth mineral. ${ }^{5}$ As a result, tooth enamel can begin to dissolve. However, when the $\mathrm{pH}$ is above this value, the saliva and plaque are supersaturated with respect to tooth mineral. The calcium and phosphate ions in saliva then start to repair any damaged mineral crystals in the enamel - the process of remineralisation.

Thus, acidic conditions contribute to bringing phosphate and hydroxyl ions below saturation levels, allowing the solid hydroxyapatite crystals of the tooth mineral to dissolve. If above saturation levels, the chemical reaction will move towards remineralisation and any damaged crystals will be repaired by the acquisition of ions from the solution. ${ }^{1}$

Stimulation of saliva flow results in an increase in the washing out of acids (and sugars), and also an increase in the amount and concentration of bicarbonate buffer and of remineralising ions. ${ }^{1}$

\section{Salivary gland disorders}

The importance of the salivary glands - and saliva - tends to go unnoticed until the glands malfunction. The consequences are severe and impact greatly on quality of life. Symptoms may start with a constant thirst, difficulty in speaking, eating, tasting and swallowing foods and progress to tooth decay and oral infections. ${ }^{6}$ The most common salivary gland disorder is xerostomia, which is the subjective feeling of dryness throughout the mouth.

Clinical studies have shown that chewing sugar-free gum stimulates the salivary glands to produce a strong flow of saliva (a 10-12 fold increase over unstimulated saliva). ${ }^{7}$ The effect of stimulation is to increase the concentration of bicarbonate in the saliva entering the mouth. This bicarbonate raises the $\mathrm{pH}$ of the saliva and greatly increases its buffering power: the saliva is, therefore, much more effective in neutralising and buffering food acids and acids arising in plaque from the fermentation of carbohydrate. ${ }^{1}$ 


\section{Figure 3: The process of tooth remineralisation}

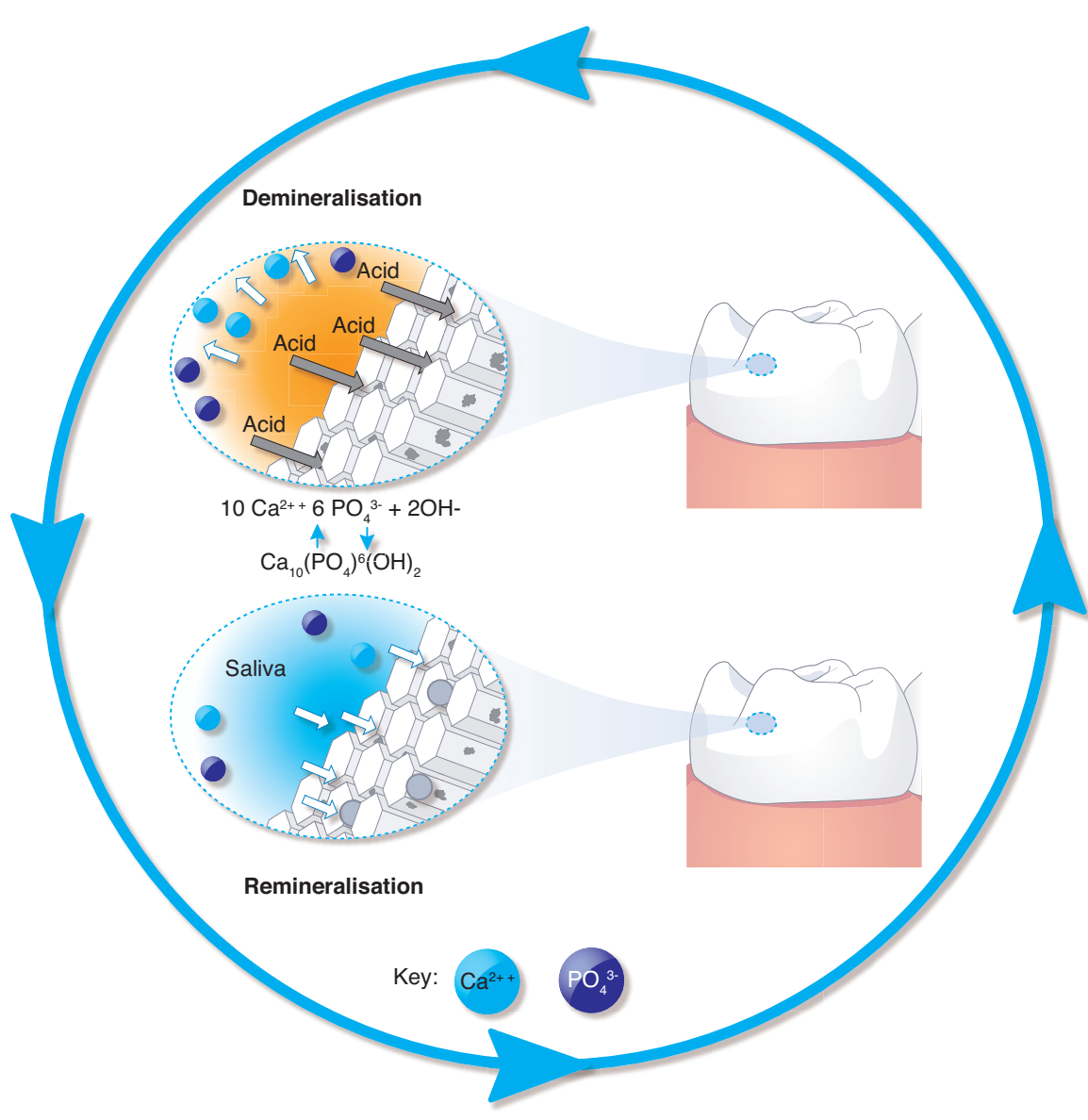

\section{'IN THE PAST, IT WAS COMMONLY BELIEVED}

\section{THAT DRY MOUTH AND DECLINING}

\section{SALIVARY FUNCTION WERE PURELY A NATURAL}

\section{CONSEQUENCE OF AGEING'}

\section{Xerostomia}

Studies conducted on outpatients and in the general population show that xerostomia affects about 1 in 4 people. ${ }^{8}$ Salivary flow rate patterns demonstrate both daily and seasonal variation, with peaks in mid-afternoon and higher flow rates in the winter than in the summer. During sleep, saliva flow rate is minimal. ${ }^{9}$ People who complain of dry mouth do not necessarily have a very low flow rate; conversely, those with a low unstimulated flow rate do not always complain of dry mouth. It is therefore of greater significance to establish Whether or not the flow rate has changed adversely in a particular individual. ${ }^{10}$

Reduced salivary flow is due to hypofunction of the salivary glands. This may be reversible, due to anxiety, acute infection, dehydration or the effects of some drugs. There are also some permanent causes of xerostomia such as congenital abnormalities, Sjögren's syndrome, HIV/ AIDS and the result of head and neck irradiation. However, xerostomia is most commonly associated with the use of xerogenic drugs. More than 400 medicines induce salivary gland hypofunction, including tricyclic antidepressants, antihistamines, certain antihypertensives and drugs with sympathomimetic actions (e.g. some bronchodilators). ${ }^{11}$

In the past, it was commonly believed that dry mouth and declining salivary function were purely a natural consequence of aging. While it is true that salivary gland dysfunctions are more prevalent in older populations, studies suggest that salivary gland dysfunction is due to a combination of ageing per se and the higher incidence of chronic illnesses and the greater use of drugs by the ageing population - both of which can impact the production of saliva. ${ }^{12}$

1. de Almeida PD, Grégio AM, Machado MA, et al. Saliva composition and functions: a comprehensive review' J Contemp Dent Pract 2008: 72-80.

2. Dawes $\mathrm{C}$ and Watanabe $\mathrm{S}$. The effect of taste adaptation on salivary flow rate and salivary sugar clearance, J Dent Res 1987: 740.

3. Featherstone JD. Dental caries: a dynamic disease process, Aust Dent J 2008: 286-291.

4. Li X, Wang J, Joiner A, Chang J. The remineralisation of enamel: a review of the literature, J Dent 2014: 12-20.

5. Hicks J, Garcia-Godoy F, Flaitz C. Biological factors in dental caries enamel structure and the caries process in the dynamic process of demineralisation and remineralisation (part 2) J Clin Pediatr Dent 2004: 119-124.

6. Ship JA. Diagnosing, managing and preventing salivary gland disorders. Oral Diseases 2002; 8, 77-89.

7. Dawes C, Macpherson LM. Effects of nine different chewing gums and lozenges on salivary flow rate and pH. Caries Res. 1992: 176-182

8. Billings RJ. Studies on the prevalence of xerostomia: preliminary results. Caries Res. 1989: 124(abstract 124).

9. Whelton $\mathrm{H}$. The anatomy and physiology of salivary glands. In: Edgar M, Dawes C, O'Mullane D, eds. Saliva and oral health. 4th edition. Bicester: Stephen Hancocks Ltd, 2012.

10. Dawes C. Factors influencing Salivary flow rate and composition. In: Edgar M, Dawes C, O'Mullane D, eds. Saliva and oral health. 4th edition. Bicester: Stephen Hancocks Ltd, 2012: 37-55.

11. Sreebny LM, Schwartz SS. A reference guide to drugs and dry mouth. Gerodontology. 1986: 75-99.

12. Atkinson JC, Fox PC. Salivary gland dysfunction. Clin Geriatr Med. 1992: 499-511.

bdjteam 2015123 